\title{
LINEAR BIJECTIONS ON VON NEUMANN FACTORS COMMUTING WITH $\lambda$-ALUTHGE TRANSFORM
}

\author{
FERNANDA BOTELHO, LAJOS MOLNÁR, AND GERGŐ NAGY \\ Dedicated to the memory of Professor James Jamison
}

\begin{abstract}
We prove that a bijective linear transformation between von Neumann factors which commutes with a $\lambda$-Aluthge transform is necessarily a nonzero scalar multiple of an algebra ${ }^{*}$-isomorphism in the case of algebras which are not of type $\mathrm{I}_{2}$. As for type $\mathrm{I}_{2}$ factors, i.e., in the particular case of the algebra of 2 by 2 complex matrices, we also present a complete description of those transformations which is a bit different. Namely, nonzero scalar multiples of algebra *-antiisomorphisms perturbed by the negative of the trace functional times the identity also show up.
\end{abstract}

\section{Introduction and Statement of the Main Result}

In this paper we present a description of bijective linear transformations between von Neumann factors which commutes with a so-called $\lambda$-Aluthge transform. We prove that (with the exception of the case of the algebra of 2 by 2 complex matrices) the only such linear transformations are the nonzero scalar multiples of algebra ${ }^{*}$-isomorphisms. Clearly, our result can also be viewed as a new characterization of algebra *isomorphisms between such algebras. The problem of studying transformations which respect the Aluthge transform originates from a question posed by Professor James Jamison. The first two authors dedicate the paper to his memory with everlasting respect and sincere friendship.

Our approach to the solution of the problem is essentially based on ideas from the theory of linear preservers. Our latest paper in this area is [18]. As for general preservers, we refer to the volume [17] of the second author and to the very recent volume [23] of Šemrl.

2010 Mathematics Subject Classification. Primary: 47B49, 46L40.

Key words and phrases. Aluthge transform, Duggal transform, isomorphisms of operator algebras.

The second author was supported by the Campus Hungary Program TÁMOP4.2.4B/2-11/1-2012-0001. The second and third authors were supported by the "Lendület" Program (LP2012-46/2012) of the Hungarian Academy of Sciences. 
Let $H$ be a complex Hilbert space and denote by $B(H)$ the algebra of all bounded linear operators on $H$. For an operator $A \in B(H)$ with polar decomposition $A=U|A|\left(U\right.$ is a partial isometry with $\left.\operatorname{ker} U=\operatorname{rng}|A|^{\perp}\right)$, the Aluthge transform $\tilde{A}$ is defined by

$$
\tilde{A}=|A|^{\frac{1}{2}} U|A|^{\frac{1}{2}}
$$

see the original source [2]. This operator transform is an important tool in the study of hyponormal operators and still very active research is going on concerning it. For demonstration we only refer to a few recent papers [1] [5], [7], [16], [19], [24] and for important former results the reader can consult e.g. [3], [4], [12], [14], [15], [25], [26].

In [20] Okubo introduced a more general notion called $\lambda$-Aluthge transform which has later been studied also in detail. This is defined for any $0<\lambda \leq 1$ by

$$
\Delta_{\lambda}(A)=|A|^{\lambda} U|A|^{1-\lambda}, \quad A \in B(H) .
$$

Clearly, for $\lambda=1 / 2$ we obtain the usual Aluthge transform. As for the case $\lambda=1$, the operator $\Delta_{1}(A)=|A| U$ is called the Duggal transform of $A \in B(H)$ and is denoted by $\hat{A}$.

The main result of the paper is the following.

Theorem 1. Let $\mathscr{A}, \mathscr{B}$ be von Neumann factors and $0<\lambda \leq 1$ a given real number. Assume $\phi: \mathscr{A} \rightarrow \mathscr{B}$ is a bijective linear transformation.

1. If $\mathscr{A}$ is not of type $I_{2}$, then we have

$$
\Delta_{\lambda}(\phi(A))=\phi\left(\Delta_{\lambda}(A)\right), \quad A \in \mathscr{A}
$$

if and only if there is an algebra ${ }^{*}$-isomorphism $\theta: \mathscr{A} \rightarrow \mathscr{B}$ and a nonzero scalar $c \in \mathbb{C}$ such that

$$
\phi(A)=c \theta(A), \quad A \in \mathscr{A} .
$$

2. If $\mathscr{A}$ is of type $I_{2}$, then $\mathscr{B}$ is also of type $I_{2}$ and without loss of generality we can assume that they both coincide with the algebra of all 2 by 2 complex matrices. Then $\phi$ satisfies (1) if and only if it is either of the form

$$
\phi(A)=c V A V^{*}, \quad A \in \mathscr{A}
$$

or of the form

$$
\phi(A)=c\left(V A^{t} V^{*}-(\operatorname{Tr} A) I\right), \quad A \in \mathscr{A},
$$

where $V$ is unitary, $c$ is a nonzero scalar, ${ }^{t}$ stands for the transpose and $\mathrm{Tr}$ stands for the usual trace functional on matrices. 


\section{Proof}

This section is devoted to the proof of the main result. First observe that for every algebra *-isomorphism $\theta: \mathscr{A} \rightarrow \mathscr{B}$ we obtain the property (1), i.e., $\theta$ commutes with the $\lambda$-Aluthge transform. Similarly, multiplication by a fixed nonzero scalar also has this property. Thus, as for the first part of our main theorem, sufficiency is immediate, we need to prove only the necessity. This will be done in several steps.

We begin with some auxiliary results. First we recall the notion of quasinormality. The operator $A \in B(H)$ is called quasinormal if it satisfies

$$
A\left(A^{*} A\right)=\left(A^{*} A\right) A .
$$

It is known (see, e.g., [10, Lemma 4.1]) that given an operator $A \in B(H)$ with polar decomposition $A=U|A|, A$ is quasinormal if and only if $U|A|=|A| U$.

The next lemma characterizes certain particular $\lambda$-Aluthge transforms.

Lemma 2. Let $0<\lambda \leq 1$ and pick any $A \in B(H)$. We have $\Delta_{\lambda}(A)=A$ if and only if $A$ is quasinormal. Moreover, $\Delta_{\lambda}(A)=0$ holds if and only if $A^{2}=0$.

Proof. Consider the polar decomposition $A=U|A|$. We have $\Delta_{\lambda}(A)=A$ if and only if

$$
\left(|A|^{\lambda} U-U|A|^{\lambda}\right)|A|^{1-\lambda}=0 .
$$

If $\lambda=1$, this is equivalent to $|A| U-U|A|=0$. If $\lambda<1$, then for every $x \in\left(\operatorname{rng}|A|^{1-\lambda}\right)^{\perp}=\operatorname{ker}|A|^{1-\lambda}=\operatorname{ker}|A|=\operatorname{ker}|A|^{\lambda}=(\operatorname{rng}|A|)^{\perp}$ we have $U x=0=|A|^{\lambda} x$ implying $\left(|A|^{\lambda} U-U|A|^{\lambda}\right) x=0$. Therefore, (2) holds if and only if $|A|^{\lambda} U-U|A|^{\lambda}=0$ which is equivalent to the commutativity of $U$ and $|A|$. But as already mentioned above, this holds exactly when $A$ is quasinormal.

The equality $\Delta_{\lambda}(A)=0$ is equivalent to $|A|^{\lambda} U|A|^{1-\lambda}=0$. Since on the subspace $\left(\mathrm{rng}|A|^{1-\lambda}\right)^{\perp}=(\mathrm{rng}|A|)^{\perp}$ the partial isometry $U$ vanishes, the latter equality holds if and only if $|A|^{\lambda} U=0$. This is equivalent to that the subspace $\operatorname{rng} U=\overline{\operatorname{rng} A}$ is contained in $\operatorname{ker}|A|^{\lambda}=\operatorname{ker}|A|=\operatorname{ker} A$ which holds exactly when $A^{2}=0$.

The next lemma gives a characterization of normal operators among quasinormal ones.

Lemma 3. Assume $A \in B(H)$ is quasinormal. Then $A$ is normal if and only if $A+\lambda I$ is quasinormal for some nonzero scalar $\lambda \in \mathbb{C}$.

Proof. The necessity is obvious. For the sufficiency suppose that we have a nonzero scalar $\lambda \in \mathbb{C}$ such that

$$
(A+\lambda I)(A+\lambda I)^{*}(A+\lambda I)=(A+\lambda I)^{*}(A+\lambda I)(A+\lambda I) .
$$


Performing the mutiplications and using the quasinormality of $A$, comparison of the two sides of the equality gives us that $\lambda\left(A^{*} A+A A^{*}\right)=$ $2 \lambda A^{*} A$ and this implies that $A$ is normal.

The next proposition shows that, under certain conditions, bijective linear transformations which preserve quasinormal elements also preserve normal elements.

Proposition 4. Let $H, K$ be complex Hilbert spaces. Let $\mathscr{A}$ be any *subalgebra of $B(H)$ containing the identity and, similarly, let $\mathscr{B}$ be any *-subalgebra of $B(K)$ containing the identity. Assume that $\mathscr{B}$ has trivial center $\mathbb{C} I$. Let $\phi: \mathscr{A} \rightarrow \mathscr{B}$ be a bijective linear map which sends normal elements to quasinormal elements. Then $\phi(I)$ is a scalar multiple of the identity and $\phi$ sends normal elements to normal elements.

Proof. We first prove that $\phi(I)$ is a scalar multiple of the identity. Pick any normal element $N$ of $\mathscr{A}$. Then $I+t N, I+i t N$ are normal for every $t \in \mathbb{R}$. Consider the identities

$$
\phi(I+t N) \phi(I+t N)^{*} \phi(I+t N)=\phi(I+t N)^{*} \phi(I+t N) \phi(I+t N)
$$

and

(4) $\phi(I+i t N) \phi(I+i t N)^{*} \phi(I+i t N)=\phi(I+i t N)^{*} \phi(I+i t N) \phi(I+i t N)$.

Using the linearity of $\phi$, computing and comparing the coefficients of the variable $t$ on both sides of the equalities (3) and (4), we have

$$
\begin{aligned}
\phi(N) \phi(I)^{*} \phi(I)+\phi(I) \phi(N)^{*} \phi(I)+\phi(I) \phi(I)^{*} \phi(N) \\
=\phi(N)^{*} \phi(I) \phi(I)+\phi(I)^{*} \phi(N) \phi(I)+\phi(I)^{*} \phi(I) \phi(N)
\end{aligned}
$$

and

$$
\begin{aligned}
\phi(N) \phi(I)^{*} \phi(I)- & \phi(I) \phi(N)^{*} \phi(I)+\phi(I) \phi(I)^{*} \phi(N) \\
& =-\phi(N)^{*} \phi(I) \phi(I)+\phi(I)^{*} \phi(N) \phi(I)+\phi(I)^{*} \phi(I) \phi(N) .
\end{aligned}
$$

Subtracting these two equalities we obtain

$$
\phi(I) \phi(N)^{*} \phi(I)=\phi(N)^{*} \phi(I) \phi(I)
$$

for every normal element $N$ of $\mathscr{A}$. Since every element of $\mathscr{A}$ is the linear combination of self-adjoint ones, we have

$$
\phi(I) \phi(X)^{*} \phi(I)=\phi(X)^{*} \phi(I) \phi(I), \quad X \in \mathscr{A} .
$$

By the surjectivity of $\phi$ we deduce

$$
\phi(I) T \phi(I)=T \phi(I) \phi(I), \quad T \in \mathscr{B} .
$$

We claim that this implies that $\phi(I)$ is a scalar multiple of the identity. 
To prove this, denote $A=\phi(I)$. We know that $A$ is quasinormal and satisfies

$$
A T A=T A^{2}
$$

for every $T \in \mathscr{B}$. Substituting $T A^{*}$ into (5) we obtain $A T A^{*} A=T A^{*} A^{2}$. Using the quasinormality of $A$, we have $T A^{*} A^{2}=T A A^{*} A$ and hence we deduce $A T A^{*} A=T A A^{*} A$. It follows that $\left(A T A^{*}-T A A^{*}\right) A=0$ and thus $A T A^{*}-T A A^{*}$ vanishes on the range of $A$. Since it clearly vanishes also on $(\operatorname{rng} A)^{\perp}=\operatorname{ker} A^{*}$, we obtain that $A T A^{*}=T A A^{*}$ holds for every $T \in \mathscr{B}$. The element $A T A^{*}$ is self-adjoint for any self-adjoint $T$, so it follows that the same holds also for $T A A^{*}$. This implies that we have $T A A^{*}=A A^{*} T$ for every self-adjoint $T \in \mathscr{B}$ and hence for any element $T$ of $\mathscr{B}$, too. This gives us that the positive element $A A^{*}$ is in the center of $\mathscr{B}$ which implies that $A A^{*}=c I$ holds for some positive scalar $c \in \mathbb{R}$ (observe that $A=\phi(I) \neq 0$ ). Substituting $A^{*} T$ into (5) we have $c T A=A A^{*} T A=A^{*} T A^{2}$. Multiplying by $A^{*}$ on the right we obtain $c^{2} T=c A^{*} T A$. Multiplying again, this time by $A$ on the left, we deduce $c^{2} A T=c^{2} T A$ which implies $A T=T A$. Since $T \in \mathscr{B}$ is arbitrary, we have that $A$ is in the center of $\mathscr{B}$ and therefore $\phi(I)$ is a nonzero scalar multiple of the identity.

To complete the proof assume that $N \in \mathscr{A}$ is normal. Then we have that $\phi(N)$ and $\phi(N)+\phi(I)=\phi(N+I)$ are quasinormal and by Lemma 3 we conclude that $\phi(N)$ is normal.

To the proof of our main result we need the following theorem which is in fact a simple corollary of Theorem 4.1 in [6] (see also Section 7.2 in the monograph [9]) that concerns normal-preserving linear maps on general prime *-algebras.

Theorem 5. Let $\mathscr{A}, \mathscr{B}$ be von Neumann factors and let $\phi: \mathscr{A} \rightarrow \mathscr{B}$ be a bijective linear map which sends normal elements to normal elements. If $\mathscr{A}$ is not of type $I_{2}$, then there is a nonzero number $c$, an algebra *isomorphism or an algebra *-antiisomorphism $\theta: \mathscr{A} \rightarrow \mathscr{B}$ and a linear functional $f: \mathscr{A} \rightarrow \mathbb{C}$ such that $\phi$ is of the form

$$
\phi(A)=c \theta(A)+f(A) I, \quad A \in \mathscr{A} .
$$

We shall apply the result above in the case of infinite von Neumann factors and for this we also need the following lemma.

Lemma 6. Assume $\mathscr{A}$ is an infinite von Neumann factor. Then $\mathscr{A}$ is linearly generated by the set of its non-normal quasinormal elements.

Proof. Let $P \in \mathscr{A}$ be a projection whose orthogonal complement $I-P$ is an infinite projection. Then we have a proper subprojection $Q$ of $I-P$ and a partial isometry $V \in \mathscr{A}$ such that $V^{*} V=I-P$ and $V V^{*}=Q$. Then one 
can easily check that $P+V, P-V$ are non-normal quasinormal elements. Observe that here $P$ can in fact be replaced by any normal element $A \in \mathscr{A}$ with the property $A=P A P$. If $I-P$ is finite, then $P$ is infinite and hence, by halving, it is the sum of two mutually orthogonal subprojections $P^{\prime}, P^{\prime \prime}$ which are equivalent to $P$ and hence are infinite. From what we have already proved it follows that $P^{\prime}+(I-P), P^{\prime \prime}-(I-P)$ are linear combinations of non-normal quasinormal elements. Therefore, every projection is in the linear span of the set of non-normal quasinormal elements. It is well-known that every element of a von Neumann factor is the finite linear combination of projections (e.g., see [13]), hence the proof is complete.

To the next proposition we recall that by Lemma 2 any linear bijection between von Neumann factors which commutes with a $\lambda$-Aluthge transform preserves the quasinormal elements in both directions.

Proposition 7. Let $\mathscr{A}, \mathscr{B}$ be von Neumann factors and let $\phi: \mathscr{A} \rightarrow \mathscr{B}$ be a bijective linear map which sends quasinormal elements to quasinormal elements. Assuming $\mathscr{A}$ is not of type $I_{2}$, there is a nonzero complex number c, an algebra ${ }^{*}$-isomorphism or an algebra ${ }^{*}$-antiisomorphism $\theta: \mathscr{A} \rightarrow \mathscr{B}$ and a linear functional $f: \mathscr{A} \rightarrow \mathbb{C}$ such that $\phi$ is of the form

$$
\phi(A)=c \theta(A)+f(A) I, \quad A \in \mathscr{A} .
$$

Assuming $\mathscr{A}$ is an infinite factor, we necessarily have that $f=0$ and that $\theta$ is an algebra *-isomorphism. Hence, in that case $\phi$ is a nonzero scalar multiple of an algebra *-isomorphism.

Proof. By Proposition $4 \phi$ sends normal elements to normal elements and Theorem 5 applies. Thus we have that there is a nonzero complex number $c$, an algebra *-isomorphism or an algebra *-antiisomorphism $\theta: \mathscr{A} \rightarrow \mathscr{B}$ and a linear functional $f: \mathscr{A} \rightarrow \mathbb{C}$ such that $\phi$ is of the form

$$
\phi(A)=c \theta(A)+f(A) I, \quad A \in \mathscr{A} .
$$

Assume now that $\mathscr{A}$ is an infinite factor. We first show that $\theta$ is not an algebra ${ }^{*}$-antiisomorphism. Suppose, on the contrary, that it is an antiisomorphism. Since $I \in \mathscr{A}$ is an infinite projection, it has a proper subprojection $P$ which is equivalent to $I$. That means that we have a partial isometry $S \in \mathscr{A}$ such that $S^{*} S=I, S S^{*}=P$. Let $\mu=f(S) / c$. Since $S$ is obviously quasinormal, it follows that

$$
\theta(S+\mu I)=\theta(S)+\mu I=(1 / c) \phi(S)
$$

is also quasinormal. Using the fact that $\theta$ is supposed to be an algebra *-antiisomorphism, we deduce

$$
(S+\mu I)(S+\mu I)^{*}(S+\mu I)=(S+\mu I)(S+\mu I)(S+\mu I)^{*} .
$$


One can easily calculate and infer that

$$
S S^{*} S+\mu S^{*} S=S S S^{*}+\mu S S^{*}
$$

implying $S+\mu I=S P+\mu P$. It follows $S(I-P)=-\mu(I-P)$. Since $S S^{*}=P$, the range of $S$ equals the range of $P$. Therefore it follows that $\mu$ is necessarily 0 from which we obtain $S=S P$. Multiplying by $S^{*}$ from the left we get $I=P$, a contradiction. Therefore, it follows that $\theta$ is an algebra *-isomomorphism.

We next show that $f=0$. Setting $\psi=(1 / c) \theta^{-1} \circ \phi: \mathscr{A} \rightarrow \mathscr{A}$, it follows that $\psi$ is a bijective linear map which sends quasinormal elements to quasinormal elements and $\psi(A)=A+g(A) I$ holds for every $A \in \mathscr{A}$, where $g=(1 / c) f$. Observe the following. If $A \in \mathscr{A}$ is quasinormal and not normal, then $g(A)=0$. Indeed, assume on the contrary that $g(A) \neq 0$. Using the quasinormality of $\psi(A)$ and Lemma 3, from $\psi(A)=A+g(A) I$ we infer that $A$ is normal, a contradiction. Therefore $g$, and hence $f$ too, vanishes on non-normal quasinormal elements. By Lemma 6 we obtain that $f=0$. This completes the proof.

Observe that the proposition above proves the necessity in the first statement of our main result in the case of infinite factors. As for the finite case, we need to take into consideration also the fact (see Lemma 2 again) that if a linear bijection commutes with a $\lambda$-Aluthge transform, then it preserves the square-zero operators in both directions.

In the proposition below, Tr stands for the unique normalized trace in a finite factor.

Proposition 8. Let $\mathscr{A}, \mathscr{B}$ be von Neumann factors and assume that $\mathscr{A}$ is finite and not of type $I_{2}$. Let $\phi: \mathscr{A} \rightarrow \mathscr{B}$ be a bijective linear map which sends quasinormal elements to quasinormal elements and preserves square-zero elements in both directions. Then there are scalars $c, d \in \mathbb{C}, c \neq 0$ and an algebra *-isomorphism or an algebra *-antiisomorphism $\theta: \mathscr{A} \rightarrow \mathscr{B}$ such that $\phi$ is of the form

$$
\phi(A)=c \theta(A)+d \operatorname{Tr}(A) I, \quad A \in \mathscr{A} .
$$

Proof. By Proposition 7 we have a nonzero scalar $c$, an algebra *isomorphism or an algebra *antiisomorphism $\theta: \mathscr{A} \rightarrow \mathscr{B}$ and a linear functional $f: \mathscr{A} \rightarrow \mathbb{C}$ such that $\phi$ is of the form

$$
\phi(A)=c \theta(A)+f(A) I, \quad A \in \mathscr{A} .
$$

In particular, it follows that $\mathscr{A}$ and $\mathscr{B}$ are *-isomorphic or *-antiisomorphic to each other which means that $\mathscr{A}$ and $\mathscr{B}$ are of the same type.

We need to show that $f$ is a scalar multiple of the trace. We assert that $f$ vanishes on the set of all square-zero elements of $\mathscr{A}$. Indeed, 
for any $A \in \mathscr{A}$ with $A^{2}=0$ we have that $\phi(A), c \theta(A)$ are square-zero elements of $\mathscr{B}$. From squaring the equation displayed in (6) we deduce $f(A)(2 c \theta(A)+f(A) I)=0$. Assuming $f(A)$ is not zero, we have that $\theta(A)$ is a scalar multiple of the identity which implies that so is $A$. Since $A$ is square-zero, we get $A=0$ and this contradicts $f(A) \neq 0$. Therefore, $f(A)=0$ holds for every square-zero $A \in \mathscr{A}$.

We claim that in any finite von Neumann factor, the linear span of the square-zero elements equals the kernel of the trace functional. Indeed, in the case of factors of type $\mathrm{II}_{1}$ we argue as follows. By Theorem 3 in [21], the linear span of square-zero elements coincides with the linear span of commutators. On the other hand, it was proved in [11] (cf. the introduction in [13]) that every trace-zero element is the sum of 10 commutators. This clearly implies our claim. If $\mathscr{A}$ is a finite factor of type I, then $\mathscr{A}$ is isomorphic to the algebra of all $n$ by $n$ matrices and our claim is wellknown to be true, see, e.g., the introduction in [8].

Therefore, we have $\operatorname{ker} \operatorname{Tr} \subset \operatorname{ker} f$ implying that $f$ is a scalar multiple of Tr and the proof is complete.

We are now in a position to present the proof of our main result.

Proof of Theorem 1. Let us agree that in the case of matrix algebras, $\mathrm{Tr}$ denotes the usual trace functional, while in the case of type $\mathrm{II}_{1}$ factors, $\mathrm{Tr}$ stands for the unique normalized trace.

We begin with the case where $\mathscr{A}$ is of type $\mathrm{I}_{2}$. Since the factors $\mathscr{A}, \mathscr{B}$ must have the same linear dimension, $\mathscr{B}$ is necessarily of the same type, so we can assume that $\mathscr{A}=\mathscr{B}=M_{2}(\mathbb{C})$. Let $\phi: M_{2}(\mathbb{C}) \rightarrow M_{2}(\mathbb{C})$ be a bijective linear transformation which commutes with the $\lambda$-Aluthge transform, i.e., $\phi$ satisfies (1) for some scalar $0<\lambda \leq 1$.

By Lemma 2 we know that $\phi$ necessarily preserves the quasinormal elements and hence, by Proposition $4, \phi(I)$ is a scalar multiple of the identity and $\phi$ sends normal elements to normal elements.

Again, using Lemma 2 we deduce that $\phi$ preserves the square-zero matrices in both directions and hence maps their linear span, i.e., the space of traceless matrices onto itself. The structure of all linear bijections on that space which preserve square-zero matrices is known due to the result Theorem 1 of Šemrl [22]. It implies that there are a nonzero scalar $c$ and a nonsingular matrix $T \in M_{2}(\mathbb{C})$ such that either we have $\phi: A \mapsto c T A T^{-1}$ or we have $\phi: A \mapsto c T A^{t} T^{-1}$ on the subspace of all traceless matrices. In the first case we deduce

$$
\begin{gathered}
\phi(A)-((\operatorname{Tr} A) / 2) \phi(I)=\phi(A-((\operatorname{Tr} A) / 2) I) \\
=c T(A-((\operatorname{Tr} A) / 2) I) T^{-1}=c T A T^{-1}-((c \operatorname{Tr} A) / 2) I
\end{gathered}
$$


i.e., $\phi(A)=c T A T^{-1}+d(\operatorname{Tr} A) I, A \in M_{2}(\mathbb{C})$ for some scalar $d$. In the second case we similarly have $\phi(A)=c T A^{t} T^{-1}+d(\operatorname{Tr} A) I, A \in M_{2}(\mathbb{C})$ for some scalar $d$.

As already mentioned, from Proposition 4 we learn that $\phi$ sends normal matrices to normal matrices meaning that $T A T^{*}$ (or $T A^{t} T^{*}$ ) is normal for every normal matrix $A$. One can easily check that the nonsingular matrix $T$ is necessarily a scalar multiple of a unitary. Since nonzero scalar multiples of algebra *-isomorphisms commute with any $\lambda$-Aluthge transform, it follows that without serious loss of generality we can assume that $\phi$ is either of the form

$$
\phi(A)=A+d(\operatorname{Tr} A) I, \quad A \in M_{2}(\mathbb{C}),
$$

or of the form

$$
\phi(A)=A^{t}+d(\operatorname{Tr} A) I, \quad A \in M_{2}(\mathbb{C}),
$$

where $d$ is a complex number. We claim that in the first case we necessarily have $d=0$, while in the second one it follows that $d=-1$.

To verify this, we will need a formula for the $\lambda$-Aluthge transform of a rank-one element of $M_{2}(\mathbb{C})$. Let $x, y \in \mathbb{C}^{2}$ be nonzero vectors such that $\|y\|=1$ and define $A=x y^{*}$, where $y^{*}$ denotes the conjugate transpose of the column vector $y$. Then

$$
\Delta_{\lambda}(A)=\langle x, y\rangle y y^{*} .
$$

Indeed, on the one hand we compute $A^{*} A=\|x\|^{2} y y^{*}$ yielding that for any number $a>0$ we have $|A|^{a}=\|x\|^{a} y y^{*}$. On the other hand, one can check that the partial isometry in the polar decomposition of $A$ is $\|x\|^{-1} x y^{*}$ and then by the definition of $\Delta_{\lambda}$ the equation (9) follows easily.

Now we are going to show that in both cases (7), (8) we have $d \in\{-1,0\}$. To see this, suppose first that we have (7) and assume on the contrary that $d \notin\{-1,0\}$. Let $x, y \in \mathbb{C}^{2}$ be linearly independent unit vectors which are not orthogonal to each other and set $A=x y^{*}$. Then

$$
\phi(A)=x y^{*}+d\langle x, y\rangle I
$$

and, since the spectrum of $x y^{*}$ is $\{0,\langle x, y\rangle\}$, it implies the invertibility of $\phi(A)$. Let $T \in M_{2}(\mathbb{C})$ be any nonsingular matrix. In this case the partial isometry in the polar decomposition of $T$ is $T|T|^{-1}$ and therefore we get that

$$
\Delta_{\lambda}(T)=|T|^{\lambda} T|T|^{-\lambda} .
$$

On the one hand, we deduce that there is an invertible self-adjoint matrix $S \in M_{2}(\mathbb{C})$ such that $\Delta_{\lambda}(\phi(A))=S \phi(A) S^{-1}$ and thus by (10) we have

$$
\Delta_{\lambda}(\phi(A))=(S x)\left(S^{-1} y\right)^{*}+d\langle x, y\rangle I .
$$


On the other hand, referring to (9) one has $\Delta_{\lambda}(A)=\langle x, y\rangle y y^{*}$ and hence

$$
\phi\left(\Delta_{\lambda}(A)\right)=\langle x, y\rangle y y^{*}+d\langle x, y\rangle I .
$$

Since $\phi$ commutes with the $\lambda$-Aluthge transform, we infer that $(S x)\left(S^{-1} y\right)^{*}=$ $\langle x, y\rangle y y^{*}$ which gives us that $S x,\langle x, y\rangle y$, resp. $S^{-1} y, y$ are scalar multiples of each other. It follows that $S x, S y$ are in the subspace of $\mathbb{C}^{2}$ generated by $y$ which contradicts the linear independence of $x, y$. Consequently, we must have $d \in\{-1,0\}$. One can apply a similar argument in the case (8) to verify that $d \in\{-1,0\}$ holds again.

In the next part we show that if $\phi$ is of the form (7), then we necessarily have $d=0$ while if $\phi$ is of the form (8) we must have $d=-1$.

If $\phi$ is of the form (8) and $d=0$, one can easily see that $\phi$ does not preserve the $\lambda$-Aluthge transform by considering $x, y$ unit vectors in $\mathbb{C}^{2}$ which are not orthogonal and $\bar{x}, \bar{y}$ are linearly independent $(-$ denotes conjugation).

Assume now that $\phi$ is of the form (7) and $d=-1$. Let $x, y \in \mathbb{C}^{2}$ be linearly independent unit vectors which are not orthogonal to each other and set $A=x y^{*}$. We will use the following notation. For $u=\left(u_{1}, u_{2}\right) \in \mathbb{C}^{2}$ let $u^{\perp}=\left(\overline{u_{2}},-\overline{u_{1}}\right)$. Clearly, $u^{\perp} \perp u$ and $\left\|u^{\perp}\right\|=\|u\|$. Using this notation, it is easy to see that $\phi(A)=\left(\left\langle x^{\perp}, y\right\rangle x-\langle x, y\rangle x^{\perp}\right)\left(x^{\perp}\right)^{*}$ yielding that

$$
\Delta_{\lambda}(\phi(A))=-\langle x, y\rangle x^{\perp}\left(x^{\perp}\right)^{*}=\langle x, y\rangle\left(x x^{*}-I\right) .
$$

On the other hand, we compute

$$
\phi\left(\Delta_{\lambda}(A)\right)=\langle x, y\rangle y y^{*}-\langle x, y\rangle I=\langle x, y\rangle\left(y y^{*}-I\right)
$$

and then it follows that $\Delta_{\lambda}(\phi(A)) \neq \phi\left(\Delta_{\lambda}(A)\right)$. This completes the proof of the necessity part of Theorem 1 in the type $\mathrm{I}_{2}$ case.

Let us now turn to the sufficiency. We need only to show that both the identity $A \mapsto A$ and the transformation $A \mapsto A-(\operatorname{Tr} A) I$ commute with any $\lambda$-Aluthge transform. Concerning the identity, there is nothing to be proven. So consider the map

$$
\phi(A)=A^{t}-(\operatorname{Tr} A) I, \quad A \in M_{2}(\mathbb{C}) .
$$

Clearly, this is a bijective linear transformation on $M_{2}(\mathbb{C})$ and we evidently have $\Delta_{\lambda}(\phi(0))=\phi\left(\Delta_{\lambda}(0)\right)$. Let $A \in M_{2}(\mathbb{C})$ be a matrix of rank 1 . There are nonzero vectors $x, y \in \mathbb{C}^{2}$ such that $A=x y^{*}$ and $\|y\|=1$. In a similar way as above, we can compute and check

$$
\Delta_{\lambda}(\phi(A))=\phi\left(\Delta_{\lambda}(A)\right)=\langle x, y\rangle\left(\bar{y} y^{t}-I\right) .
$$

So it remains to verify that $\Delta_{\lambda}(\phi(A))=\phi\left(\Delta_{\lambda}(A)\right)$ holds for any invertible matrix $A \in M_{2}(\mathbb{C})$. Using singular value decomposition, we deduce that any such matrix is unitarily similar to a matrix of the form $U D$ where $D \in M_{2}(\mathbb{C})$ is a nonsingular diagonal matrix and $U \in M_{2}(\mathbb{C})$ is unitary. 
It is clear that those products are exactly the matrices $A \in M_{2}(\mathbb{C})$ whose column vectors are nonzero and mutually orthogonal which yields that they are precisely the elements of $M_{2}(\mathbb{C})$ with columns $y, \alpha y^{\perp}$, where $y \in \mathbb{C}^{2}, \alpha \in \mathbb{C}$ are nonzero. Let $A \in M_{2}(\mathbb{C})$ be such a matrix, i.e., assume that we have numbers $y_{1}, y_{2}, \alpha \in \mathbb{C}$ for which

$$
A=\left(\begin{array}{ll}
y_{1} & \alpha \overline{y_{2}} \\
y_{2} & -\alpha \overline{y_{1}}
\end{array}\right)
$$

and $\alpha \neq 0,\left(y_{1}, y_{2}\right) \neq(0,0)$. On the one hand, since $A^{*} A$ is diagonal, using the formula (11) we easily compute

$$
\phi\left(\Delta_{\lambda}(A)\right)=\left(\begin{array}{ll}
\alpha \overline{y_{1}} & |\alpha|^{\lambda} y_{2} \\
\alpha|\alpha|^{-\lambda \overline{y_{2}}} & -y_{1}
\end{array}\right) .
$$

On the other hand,

$$
\phi(A)=\left(\begin{array}{ll}
\alpha \overline{y_{1}} & y_{2} \\
\alpha \overline{y_{2}} & -y_{1}
\end{array}\right)
$$

and hence $\phi(A)$ is invertible and $\phi(A)^{*} \phi(A)$ is diagonal. So, similarly as above, we can determine $\Delta_{\lambda}(\phi(A))$ and conclude that $\phi\left(\Delta_{\lambda}(A)\right)=$ $\Delta_{\lambda}(\phi(A))$. Since any invertible element of $M_{2}(\mathbb{C})$ is unitary similar to a matrix of the form (12), we deduce that the equality $\phi\left(\Delta_{\lambda}(A)\right)=\Delta_{\lambda}(\phi(A))$ holds for all nonsingular matrices $A \in M_{2}(\mathbb{C})$. This completes the sufficiency part of our main statement in the case of type $\mathrm{I}_{2}$ algebras.

The statement of the theorem in the case where $\mathscr{A}$ is an infinite factor follows from Proposition 7.

It remains to consider the necessity part of the theorem in the cases where $\mathscr{A}$ is of one of the types $\mathrm{II}_{1}$ or $\mathrm{I}_{n}, n \geq 3$. Let $\phi: \mathscr{A} \rightarrow \mathscr{B}$ be a bijective linear transformation which satisfies (1). By Proposition 8 we have that there are scalars $c, d \in \mathbb{C}, c \neq 0$ and an algebra *-isomorphism or an algebra *-antiisomorphism $\theta: \mathscr{A} \rightarrow \mathscr{B}$ such that $\phi$ is of the form $\phi(A)=c \theta(A)+d \operatorname{Tr}(A) I, A \in \mathscr{A}$. As already mentioned several times, multiplication by a nonnegative scalar commutes with any $\lambda$-Aluthge transform, so we may and do assume that $c=1$, i.e., that we have

$$
\phi(A)=\theta(A)+d \operatorname{Tr}(A) I, \quad A \in \mathscr{A} .
$$

We show that $d=0$ and $\theta$ is an algebra *-isomorphism.

It has also been mentioned that, since there is an algebra *-isomorphism or an algebra *-antiisomorphism between $\mathscr{A}$ and $\mathscr{B}$, they are of the same type. So, in the type I case we may assume that $\mathscr{A}=\mathscr{B}=M_{n}(\mathbb{C})$ with $n \geq 3$. Using the well-known structure of algebra *-isomorphisms and algebra *-antiisomorphisms of the full matrix algebra, we may assume that $\phi$ is either of the form

$$
\phi(A)=A+d(\operatorname{Tr} A) I, \quad A \in M_{n}(\mathbb{C}),
$$


or of the form

$$
\phi(A)=A^{t}+d(\operatorname{Tr} A) I, \quad A \in M_{n}(\mathbb{C}) .
$$

It requires only elementary reasoning to see that since $\phi$ commutes with the $\lambda$-Aluthge transform, it implies that the corresponding map $A \mapsto$ $A+d(\operatorname{Tr} A) I$ or $A \mapsto A^{t}+d(\operatorname{Tr} A) I$ of the variable $A \in M_{2}(\mathbb{C})$ necessarily commutes with the $\lambda$-Aluthge transform, too. By the first part of the proof this gives us that in the first case we have $d=0$ meaning that $\phi$ is the identity while in the second case we have $d=-1$ meaning that the transformation $A \mapsto A^{t}-(\operatorname{Tr} A) I$ on $M_{n}(\mathbb{C})$ commutes with a $\lambda$-Aluthge transform. In the first case we are done, so assume that we have the latter possibility. Then the square of the transformation $A \mapsto A^{t}-(\operatorname{Tr} A) I$ also commutes with the $\lambda$-Aluthge transform. This square is the map $A \mapsto A+(n-2)(\operatorname{Tr} A) I$. Again, considering it as a function of the variable $A \in M_{2}(\mathbb{C})$ we obtain a transformation on $M_{2}(\mathbb{C})$ which commutes with the $\lambda$-Aluthge transform. We already know that this can happen only if $n-2=0$, a contradiction. Therefore, it follows that the only bijective linear maps on $M_{n}(\mathbb{C})$ which commute with the $\lambda$-Aluthge transform are the nonzero scalar multiples of algebra *-automorphisms.

Finally, let us consider the case where $\mathscr{A}$ and hence also $\mathscr{B}$ are of type $\mathrm{II}_{1}$. Embed $M_{n}(\mathbb{C})$ into $\mathscr{A}$ by which we mean the following. Choose mutually equivalent pairwise orthogonal projections $P_{1}, \ldots, P_{n}$ in $\mathscr{A}$ with sum I. Pick partial isometries $V_{i j}$ with $V_{i j} V_{i j}^{*}=P_{i}$ and $V_{i j}^{*} V_{i j}=P_{j}, i, j \in$ $\{1, \ldots, n\}$. Select one $k_{0}$ from $\{1, \ldots, n\}$ and define $E_{i j}=V_{i k_{0}} V_{j k_{0}}^{*}$ for all $i, j \in\{1, \ldots, n\}$. Then the elements $E_{i j} \in \mathscr{A}$ form a so-called collection of matrix units corresponding to the system $P_{1}, \ldots, P_{n}$ which means that the $E_{i j}$ 's are partial isometries satisfying the following relations: $E_{i j} E_{i j}^{*}=P_{i}$, $E_{i j}^{*} E_{i j}=P_{j}, E_{i i}=P_{i}, E_{i j}^{*}=E_{j i}, E_{i j} E_{k l}=\delta_{j k} E_{i l}$ for all $i, j, k, l \in\{1, \ldots, n\}$, where $\delta_{j k}$ is the Kronecker symbol. It is apparent that

$$
\iota:\left(\lambda_{i j}\right)_{i j} \mapsto \sum_{i, j} \lambda_{i j} E_{i j}
$$

defines an algebra *-isomorphism from $M_{n}(\mathbb{C})$ onto the von Neumann subalgebra $\mathscr{A}_{n}$ of $\mathscr{A}$ generated by those matrix units. We have two possibilities for $\theta$. Assume first that it is an algebra *-isomorphism. Then the elements $Q_{i}=\theta\left(P_{i}\right)$ are mutually equivalent pairwise orthogonal projections with sum $I$ and corresponding matrix units $F_{i j}=\theta\left(E_{i j}\right), i, j \in$ $\{1, \ldots, n\}$. Let the generated von Neumann subalgebra of $\mathscr{B}$ be denoted by $\mathscr{B}_{n}$. Define

$$
\kappa: \sum_{i, j} \lambda_{i j} F_{i j} \mapsto\left(\lambda_{i j}\right)_{i j}
$$

for all $\left(\lambda_{i j}\right)_{i j} \in M_{n}(\mathbb{C})$. Since any *-algebra isomorphism between von Neumann algebras commute with the $\lambda$-Aluthge transform, we obtain 
that the linear bijection $\psi=\kappa \circ \phi_{\mathscr{A}_{n}} \circ \iota$ also has this property. Assuming $n \geq 3$, it follows that this transformation is a nonzero scalar multiple of an algebra *-isomorphism. On the other hand, by (13) we have $\psi(I)=$ $(1+d) I$ which implies that the corresponding scalar is necessarily $1+d$. We deduce that

$$
A \mapsto \frac{1}{1+d}(\theta(A)+d(\operatorname{Tr} A) I)
$$

is an algebra *-isomorphism on $\mathscr{A}_{n}$. In particular, the image of $P_{1}$ is a projection. Simple computation using $\operatorname{Tr} P_{1}=1 / n$ shows that this can happen in the case $n \geq 3$ only if $d=0$.

Assume now that $\theta$ is an algebra *-antiisomorphism. Define $Q_{i}=\theta\left(P_{i}\right)$ and $F_{i j}=\theta\left(E_{i j}\right)^{*}=\theta\left(E_{j i}\right), i, j \in\{1, \ldots, n\}$. Clearly the $F_{i j}$ 's form a system of matrix units corresponding to the collection $Q_{1}, \ldots, Q_{n}$ of mutually orthogonal pairwise equivalent projections with sum $I$. Let the generated von Neumann subalgebra of $\mathscr{B}$ be denoted by $\mathscr{B}_{n}^{\prime}$. Again, the transformation $\psi=\kappa \circ \phi_{\mathscr{L}_{n}} \circ \iota$ (here $\kappa$ is defined on $\mathscr{B}_{n}^{\prime}$ ) commutes with the $\lambda$ Aluthge transform and, consequently, it is a nonzero scalar multiple of an algebra *-isomorphism. As above, we obtain that this scalar is $1+d$ and then deduce that $d=0$. We then arrive to the contradiction that, on the one hand, $\psi$ is an algebra *-isomorphism while, on the other hand, $\theta$ is an algebra *-antiisomorphism.

This completes the proof of our main theorem.

\section{REFERENCES}

1. A. Abu Omar and F. Kittaneh, A numerical radius inequality involving the generalized Aluthge transform, Studia Math. 216 (2013), 69-75.

2. A. Aluthge, On $p$-hyponormal operators for $0<p<1$, Integral Equations Operator Theory 13 (1990), 307-315.

3. T. Ando, Aluthge transforms and the convex hull of the eigenvalues of a matrix, Linear and Multilinear Algebra 52 (2004), 281-292.

4. T. Ando and T. Yamazaki, The iterated Aluthge transforms of a 2-by-2 matrix converge, Linear Algebra Appl. 375 (2003), 299-309.

5. J. Antezana, E.R. Pujals and D. Stojanoff, The iterated Aluthge transforms of a matrix converge, Adv. Math. 226 (2011), 1591-1620.

6. K.I. Beidar, M. Brešar, M.A. Chebotar and Y. Fong, Applying functional identities to some linear preserver problems, Pacific J. Math. 204 (2002), 257-271.

7. F. Botelho and J. Jamison, Elementary operators and the Aluthge transform, Linear Algebra Appl. 432 (2010), 275-282.

8. P. Botta, S. Pierce and W. Watkins, Linear transformations that preserve the nilpotent matrices, Pacific J. Math. 104 (1983), 39-46.

9. M. Brešar, M.A. Chebotar and W.S. Martindale III, Functional Identities, Birkhäuser, 2007.

10. A. Brown, On a class of operators, Proc. Amer. Math. Soc. 4(1953), 723-728.

11. Th. Fack and P. de la Harpe, Sommes de commutateurs dans les algébres de von Neumann finies continues, Ann. Inst. Fourier (Grenoble) 30 (1980), 49-73. 
12. C. Foias, I. Jung, E. Ko and C. Pearcy, Completely contractivity of maps associated with Aluthge and Duggal Transforms, Pacific J. Math. 209 (2003), 249-259.

13. S. Goldstein and A. Paszkiewicz, Linear combinations of projections in von Neumann algebras, Proc. Amer. Math. Soc. 116 (1992), 175-183.

14. I. Jung, E. Ko and C. Pearcy, Aluthge transform of operators, Integral Equations Operator Theory 37 (2000), 437-448.

15. I. Jung, E. Ko and C. Pearcy, The iterated Aluthge transform of an operator, Integral Equations Operator Theory 45 (2003), 375-387.

16. S.H. Lee, W.Y. Lee and J. Yoon, Subnormality of Aluthge transforms of weighted shifts, Integral Equations Operator Theory 72 (2012), 241-251.

17. L. Molnár, Selected Preserver Problems on Algebraic Structures of Linear Operators and on Function Spaces, Lecture Notes in Mathematics, Vol. 1895, p. 236, Springer, 2007.

18. L. Molnár, Linear maps on observables in von Neumann algebras preserving the maximal deviation, J. London Math. Soc. 81 (2010), 161-174.

19. M.S. Moslehian and S.M.S. Nabavi Sales, Fuglede-Putnam type theorems via the Aluthge transform, Positivity 17 (2013), 151-162.

20. K. Okubo, On weakly unitarily invariant norm and the Aluthge transformation, Linear Algebra Appl. 371 (2003), 369-375.

21. C. Pearcy and D. Topping, Commutators and certain $I I_{1}$-factors, J. Funct. Anal. 3 (1969), 69-78.

22. P. Šemrl, Linear mappings preserving square-zero matrices, Bull. Austral. Math. Soc. 48 (1993), 365-370.

23. P. Šemrl, The optimal version of Hua's fundamental theorem of geometry of rectangular matrices, Mem. Amer. Math. Soc. 232 (2014), no. 1089.

24. J. Trepkowski, Aluthge transforms of weighted shifts on directed trees, J. Math. Anal. Appl. 425 (2015), 886-899.

25. P.Y. Wu, Numerical range of Aluthge transform of operator, Linear Algebra Appl. 357 (2002), 295-298.

26. T. Yamazaki, An expression of the spectral radius via Aluthge transformation, Proc. Amer. Math. Soc. 130 (2002), 1131-1137.

Department of Mathematical Sciences, The University of Memphis, MemPHIS, TN 38152, USA

E-mail address: mbotelho@memphis . edu

MTA-DE “Lendület” Functional Analysis Research Group, Institute of Mathematics, University of Debrecen, H-4010 Debrecen, P.O. Box 12, Hungary

E-mail address: molnarl@science. unideb.hu

URL: http://neumann.math. unideb.hu/ molnarl/

MTA-DE “Lendület” Functional Analysis Research Group, Institute of Mathematics, University of Debrecen, H-4010 Debrecen, P.O. Box 12, Hungary E-mail address: nagyg@science.unideb.hu 